\title{
The expanding phenotype of COL4A1 and COL4A2 mutations: clinical data on 13 newly identified families and a review of the literature
}

\author{
Marije E.C. Meuwissen, MD, PhD ${ }^{1,2}$, Dicky J.J. Halley, PhD', Liesbeth S. Smit, MD, \\ Maarten $\mathrm{H}$. Lequin, $\mathrm{MD}$, $\mathrm{PhD}^{4}$, Jan $\mathrm{M}$. Cobben, $\mathrm{MD}, \mathrm{PhD}^{5}$, René de Coo, $\mathrm{MD}, \mathrm{PhD}^{3}$, \\ Jeske van Harssel, MD${ }^{6}$, Suzanne Sallevelt, MD, Gwendolyn Woldringh, MD, $\mathrm{PhD}^{8}$, \\ Marjo S. van der Knaap, MD, PhD ${ }^{9}$, Linda S. de Vries, MD, PhD ${ }^{10}$ and Grazia M.S. Mancini, MD, PhD ${ }^{1}$
}

Two proal(IV) chains, encoded by COL4A1, form trimers that contain, in addition, a proa2(IV) chain encoded by COL4A2 and are the major component of the basement membrane in many tissues. Since 2005, COL4A1 mutations have been known as an autosomal dominant cause of hereditary porencephaly. COL4A1 and COL4A2 mutations have been reported with a broader spectrum of cerebrovascular, renal, ophthalmological, cardiac, and muscular abnormalities, indicated as "COL4A1 mutation-related disorders." Genetic counseling is challenging because of broad phenotypic variation and reduced penetrance. At the Erasmus University Medical Center, diagnostic DNA analysis of both COL4A1 and COL4A2 in 183 index patients was performed between 2005 and 2013. In total, 21 COL4A1 and 3 COL4A2 mutations were identified, mostly in children with porencephaly or other patterns of parenchymal hemorrhage, with a high de novo mutation rate of $40 \%(10 / 24)$. The observations in 13 novel families harboring either COL $4 A 1$ or COL4A2 mutations prompted us to review the clinical spectrum. We observed recognizable phenotypic patterns and propose a screening protocol at diagnosis. Our data underscore the importance of COL4A1 and COL4A2 mutations in cerebrovascular disease, also in sporadic patients. Follow-up data on symptomatic and asymptomatic mutation carriers are needed for prognosis and appropriate surveillance.

Genet Med advance online publication 26 February 2015

Key Words: cerebral hemorrhage; COL4A1; COL4A2; familial porencephaly; phenotype

\section{INTRODUCTION}

Since the 1980s, investigators have described the recurrence of hemorrhagic stroke within families, manifesting as porencephalic cavities on computed tomography or magnetic resonance imaging (MRI). ${ }^{1-6}$ Before these observations, the presence of congenital porencephaly, a cyst that communicates with the lateral ventricle and is (usually) seen following parenchymal hemorrhage, had often been considered the result of an external insult, for example, postanoxic perinatal bleeding without genetic substrate and, in the absence of a coagulopathy, with low risk of recurrence. Only in 2005, after observations of mouse models, were COL4A1 mutations discovered as a cause of porencephaly, with an apparent autosomal dominant inheritance. ${ }^{7-9}$ Over the years it became clear that disorders of additional organs can result from mutations in this gene. The disorder related to COL4A1 mutations is now known as a systemic disease, including a broad spectrum of cerebrovascular lesions: porencephaly and transmantle lesions, causing hydranencephaly or schizencephaly; lesions of the kidneys, leading to nephrosis and hematuria; lesions of the eyes, causing cataract, microphthalmia, and blindness; lesions of the heart, causing arrhythmia; and lesions of the skeletal muscles, causing dystrophic changes, weakness, and myoglobinuria. ${ }^{10-22}$

COL4A1 and COL4A2 encode proa1(IV) and proa2(IV) chains, respectively, which assemble to form a heterotrimeric helix with a constant 2:1 ratio (proal(IV)) $)_{2}(\operatorname{pro\alpha } 2$ (IV)). This type IV collagen is a component of nonfibrillary collagen, a main constituent of the basement membranes of many tissues, among them vascular endothelia. COL4A1 and COL4A2 consist of three domains. The amino terminal 7S domain plays an important role in intermolecular cross-linking and macromolecular organization. The triple helical domain consists of a triple amino acid repeat sequence, Gly-Xaa-Yaa, where Xaa and Yaa can be any residue, although proline is the most frequent amino acid at the Yaa position. This sequence has several interruptions containing cysteine residues, which are important

\footnotetext{
${ }^{1}$ Department of Clinical Genetics, Erasmus University Medical Center, Rotterdam, The Netherlands; ${ }^{2}$ Department of Medical Genetics, University Hospital Antwerp, Antwerp, Belgium; ${ }^{3}$ Department of Neurology, Division of Pediatric Neurology, Child Neurology, Erasmus University Medical Center, Rotterdam, The Netherlands; ${ }^{4}$ Department of Radiology, Erasmus University Medical Center, Rotterdam, The Netherlands; ${ }^{5}$ Department of Pediatric Genetics, Academic Medical Center, Amsterdam, The Netherlands; ${ }^{6}$ Department of Clinical Genetics, University Medical Center, University of Utrecht, Utrecht, The Netherlands; ${ }^{7}$ Department of Clinical Genetics, Maastricht University Medical Center, Maastricht, The Netherlands; ${ }^{8}$ Department of Clinical Genetics, University Medical Center Nijmegen, Nijmegen, The Netherlands; ${ }^{9}$ Department of Child Neurology, VU Medical Center, Amsterdam, The Netherlands; ${ }^{10}$ Department of Neonatology, University Medical Center, University of Utrecht, Utrecht, The Netherlands. Correspondence: Grazia M.S. Mancini (g.mancini@erasmusmc.nl)
} 
in giving flexibility to the collagen IV network and providing possible binding sites for intermolecular cross-linking. The C-terminal NC1 domain is important in the initiation of triple helix formation..$^{23-25}$

Mice harboring heterozygous mutations in Col4a 1 or Col4a 2 suffer from hemorrhage in the eye, brain, and skin, which already occur during gestation and sometimes lead to developmental defects of the eye and brain. Therefore, similarities in phenotypes between COL4A1 and COL4A2 mutations were to be expected. In 2012 several parallel genetic, epidemiologic, and functional studies revealed COL4A2 mutations in both familial and sporadic porencephaly. ${ }^{26-28}$

The disease resulting from both COL $4 A 1$ and COL4A2 mutations is extremely variable, with broad intra- and interfamilial variation and evidence for reduced penetrance. Sporadic individuals with severe presentation may harbor a de novo mutation.

At the Department of Clinical Genetics of the Erasmus University Medical Center in Rotterdam, the Netherlands, sequence analysis of both COL4A1 and COL4A2 has been offered in a diagnostic setting since 2005 . This review summarizes the clinical and genetic data of mutations newly identified in the past 8 years. Although many reports of the mutation spectrum have been published, no clear recent guidelines regarding genetic counseling and management of affected patients exist. Here we provide an up-to-date overview of the genotypic and phenotypic spectrum to improve clinical management and surveillance guidelines.

\section{MATERIALS AND METHODS}

At the Clinical Genetics Laboratory of the Erasmus University Medical Center in Rotterdam, 183 index patients (and whenever available both parents), mostly with cerebral hemorrhage or porencephaly, were referred for testing of COL4A1 (NM_001845.4) and COL4A2 (NM_001846.2). This was done by Sanger sequencing or by applying a next-generation sequencing panel encompassing 87 genes for brain developmental disorders, including genes for porencephaly and Aicardi-Goutières syndrome (capturing exons and intron-exon boundaries by eArray Sure Select (Agilent, Santa Clara, CA) and sequencing on the MiSeq platform (paired-end, $150 \mathrm{bp}$; Illumina, San Diego, CA), followed by confirmation of mutations with Sanger sequencing). A total of 21 COL4A1 and 3 COL4A2 putative pathogenic genomic variants were identified between 2005 and June 2013. We previously reported two of the COL4A2 and nine of the COL4A1 mutations. ${ }^{7,12,15,27,29}$ From the families with the remaining 13 novel mutations, clinical data were collected using a questionnaire sent to the referring physicians according to the institutional review board regulations of our center.

Furthermore, we reviewed the literature, focusing on the clinical phenotypes of COL4A1 and COL4A2 mutations. For this, a PubMed search was performed, identifying 27 articles with clinical and mutation data on COL4A $1^{7,8,10-22,29-40}$ and 3 articles with data on COL4A2 mutations. ${ }^{26-28} \mathrm{~A}$ total of 137 individuals with a COL4A1 mutation from 60 families and 15 individuals with a COL4A2 mutation from 7 families have been reported. Several clinical phenotypes were identified and described in more detail. In addition, both neurologic and systemic features and brain MRI findings were available for review.

\section{RESULTS}

\section{Mutation data on 13 newly identified families}

Most COL4A1 and COL4A2 mutations reported in the literature are missense changes leading to a substitution of a glycine in the Gly-Xaa-Yaa triple helical domain. In analogy to other collagenopathies, these mutations are predicted to be pathogenic and are assumed to have a dominant-negative effect. In addition, splice-site mutations led to haploinsufficiency, and frameshift mutations have been reported, indicating that haploinsufficiency of either COL4A1 or COL4A2 is another pathogenic mechanism ${ }^{27,38}$ (Figure 1). COL4A1 and COL4A2 sequence variants in our cohort were considered pathogenic when de novo, when truncating, or when previously described and were proven to be pathogenic by de novo occurrence or functional studies. Most missense changes that were either predicted (ALAMUT package, $\boldsymbol{h t t p}$ ://www.interactive-biosoftware.com) or proven to be pathogenic disrupted the Gly-XaaYaa repeat of the triple helix.

In total, 21 COL4A1 mutations, 12 of them novel, and 3 COL4A2 mutations were identified. All the known COL4A1 and COL4A2 mutations, including those reported here, are depicted in Figure 1. The pedigrees of the newly identified families are shown in Supplementary Figure S1 online.

Of the 12 cases associated with novel COL4A1 mutations, 5 were sporadic and de novo (Supplementary Figure S1 online; families D, E, H, I, and K) and 7 were familial. Of the seven familial mutations, the one in family B occurred de novo in patient I.1. Of the mutations in Supplementary Figure S1 online, only the COL4A2 mutation in family $\mathrm{L}$ was previously reported in a sporadic adult patient with intracerebral hemorrhage. ${ }^{26}$ It introduces a glycine at the triple helical domain of the protein and was shown to be pathogenic using functional studies. ${ }^{26}$ All but one of the COL4A1 mutation missense changes led to substitution of glycine in the triple helical domain of the protein (families A, B, C, G, H, I, K, and M). The changes in families A, C, G, and M have an official status of "variant of unknown clinical significance," although they are likely pathogenic in view of their predicted effect on the protein. In addition, segregation with the phenotype has been observed in families A and C. In family E, a de novo missense mutation was present, affecting the $\mathrm{NCl}$ domain. A de novo splice site mutation was identified in family $\mathrm{D}$, and family $\mathrm{G}$ harbored an inherited frameshift mutation.

\section{Clinical phenotypes identified in our cohort and in the literature}

A total of 183 index patients were tested for COL4A1 and COL4A2 mutations. A diagnosis was possible in 24 of 183 of index patients (13\%; published and unpublished), of which 10/24 (42\%) were de novo. We identified 60 COL4A1 mutation 

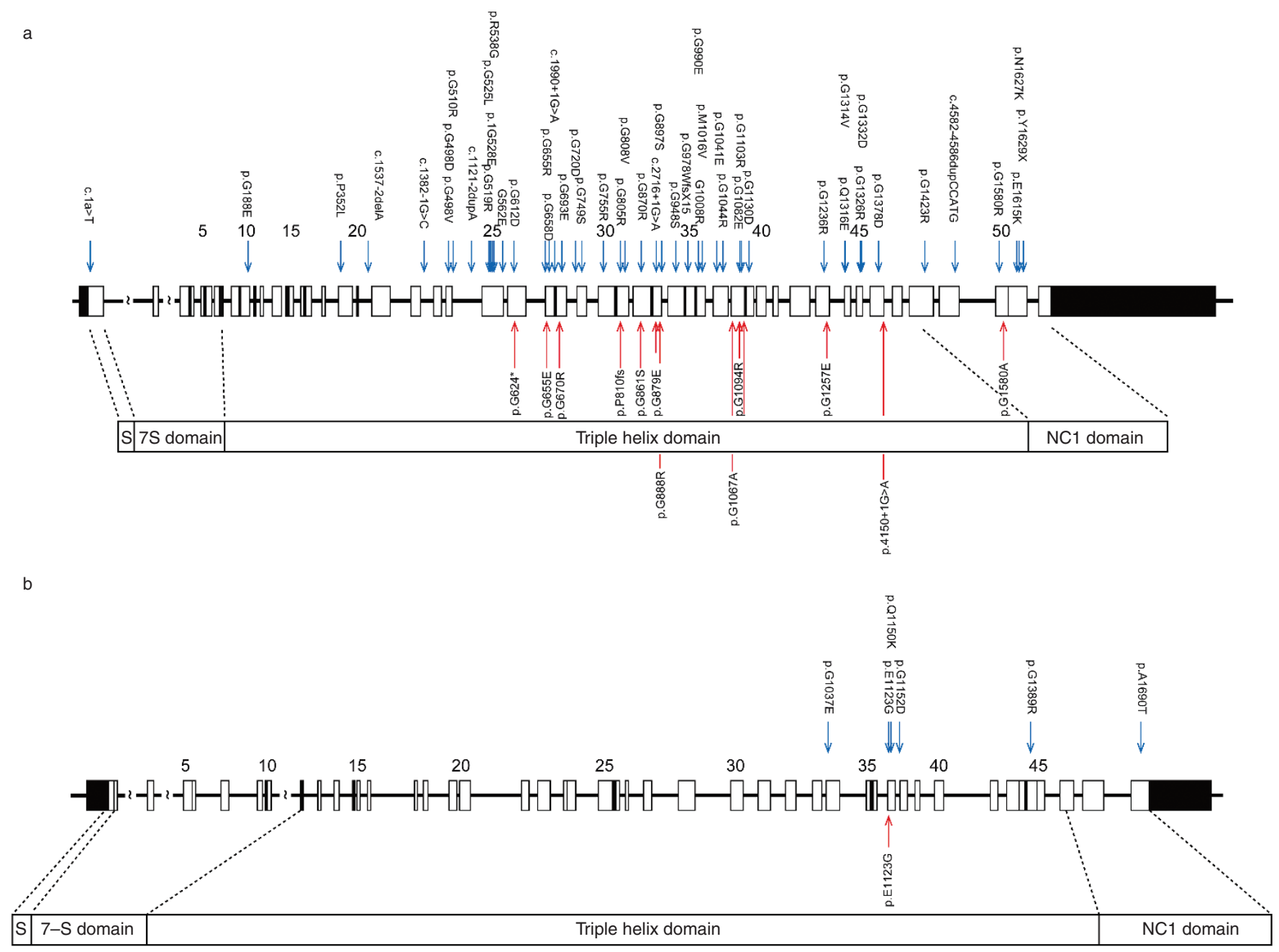

Figure 1 COL4A1 and COL4A2 mutation spectrum. The COLAA1 mutations (a) and COL4A2 mutation (b) in our novel families are depicted in the bottom panel (red arrows) and the mutations reported in the literature are depicted in the top panel (blue arrows).

families reported in the literature, of which 30 had an inherited mutation (50\%); in 16 a de novo mutation was identified (27\%), and in 14 cases $(23 \%)$ the data on parental sequencing were lacking. For COL4A2 mutations, seven families were identified, of which three had an inherited mutation, one mutation was de novo, and in three cases data on parental carrier status were lacking.

The 12 novel COL4A1 families and 1 COL4A2 family in the present cohort comprise 21 individuals with a (suspected) pathogenic mutation. Of these, five (24\%) developed signs antenatally, eight (38\%) presented with signs soon after birth, three (14\%) developed signs at a later age, and three (14\%) have been clinically asymptomatic until now. Clinical data at onset are lacking for two patients (family G). Brain MRI has not been performed in these asymptomatic carriers until now, however, and brain damage cannot be fully excluded. Seizures were the most common clinical symptom, present in nine patients (43\%). Also, a high percentage of motor dysfunction was present: hemiparesis in six (29\%) and tetraparesis in three (14\%). Developmental delay was present in eight cases (38\%).
The clinical phenotypes and mutations of the newly identified families are summarized in Table 1. A summary of the brain MRI findings, associated ophthalmological, renal, cardiac, and muscular findings, as well as incidentally reported findings in both our patients and those from the literature is provided in Table 2. We provide an overview of several phenotypes observed in our cohort. The reported data on these phenotypes in the literature are discussed later, together with additionally reported phenotypes.

\section{Prenatal and neonatal intracerebral hemorrhage and porencephaly}

Porencephaly has been reported as a result of both COL4A1 and COL4A2 mutations, most often caused by germinal matrix hemorrhage leading to deep venous infarction with subsequent tissue necrosis and porencephalic cavitation. The first reports suggested an onset around birth, ${ }^{8}$ whereas later reports also describe patients with an onset during late pregnancy. 12,21,29

In our cohort, 12 patients from 10 families $(A, B, D, E, G, H$, I, J, K, M) presented with early intracerebral hemorrhage and 

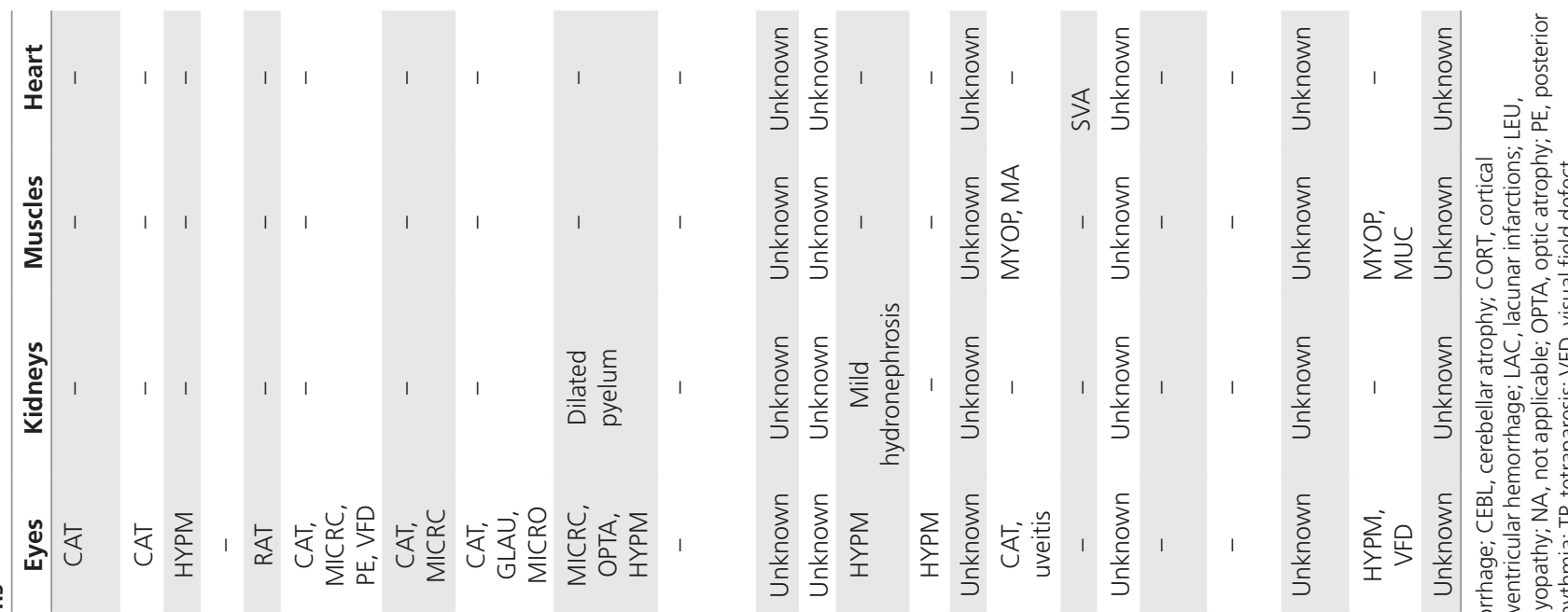

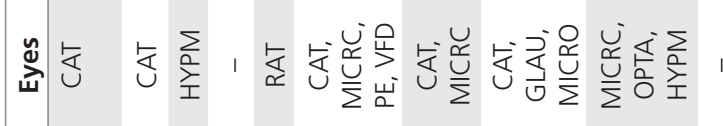

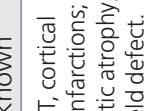

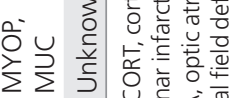

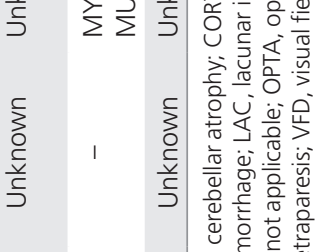

ฝे

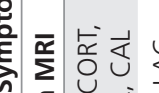

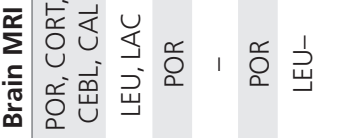

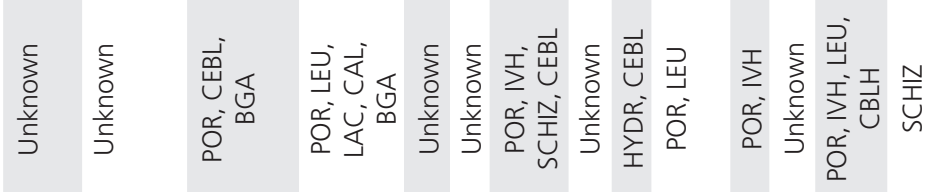

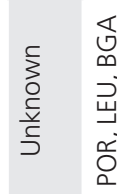

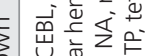

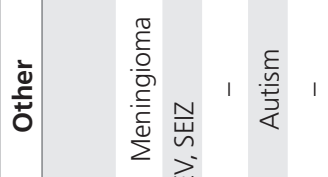

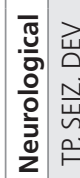

乐产, 起 ,

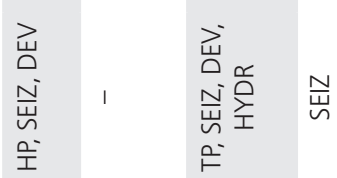

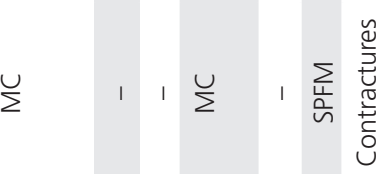

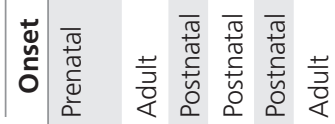

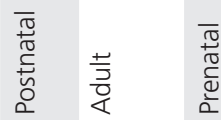

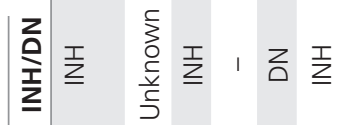

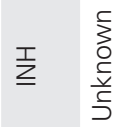

in

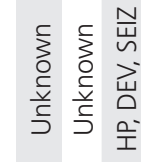

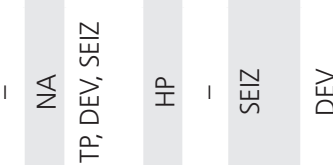

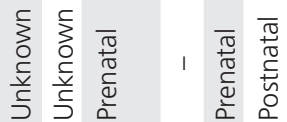

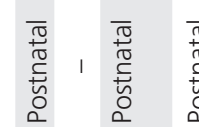

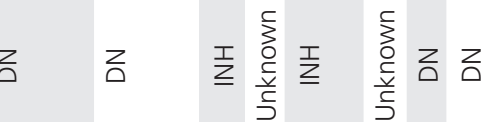

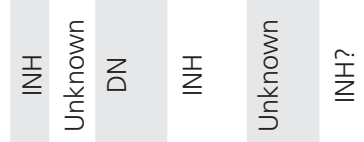

$\frac{8}{8}$

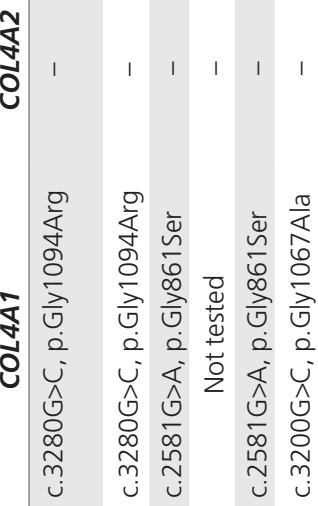

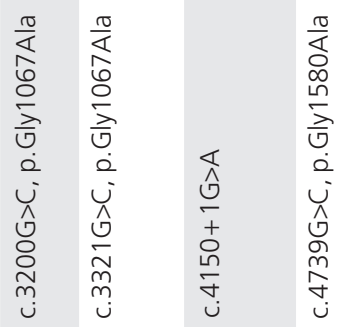

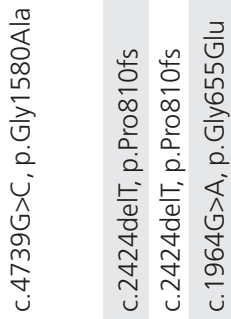

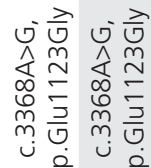

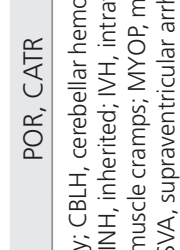

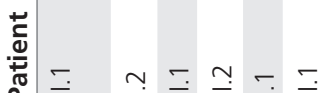

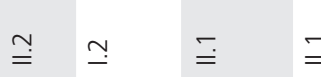

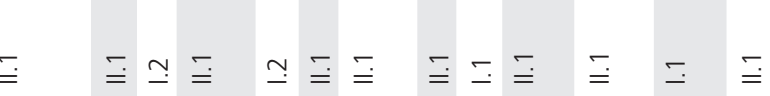

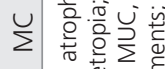

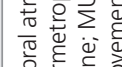

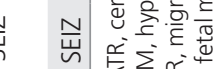

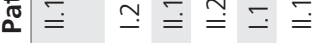

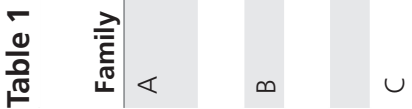


Table 2 Radiological findings and systemic findings in the literature and this patient cohort

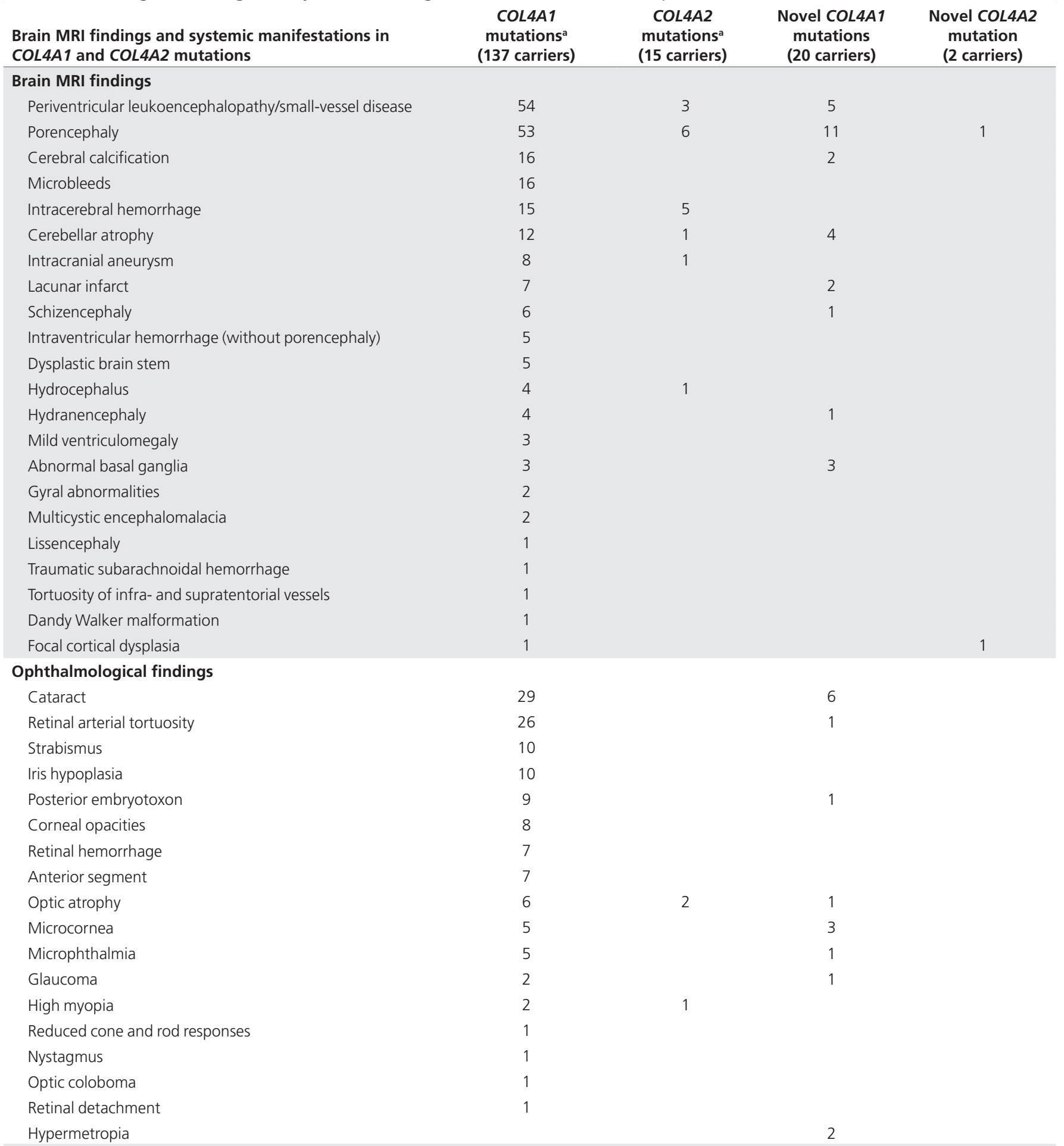

\section{Renal findings}

Renal cysts 
Table 2 Continued

\begin{tabular}{|c|c|c|c|c|}
\hline $\begin{array}{l}\text { Brain MRI findings and systemic manifestations in } \\
\text { COL4A1 and COL4A2 mutations }\end{array}$ & $\begin{array}{c}\text { COL4A1 } \\
\text { mutations }^{\mathrm{a}} \\
\text { (137 carriers) }\end{array}$ & $\begin{array}{c}\text { COL4A2 } \\
\text { mutations } \\
\text { (15 carriers) }\end{array}$ & $\begin{array}{c}\text { Novel COL4A1 } \\
\text { mutations } \\
(20 \text { carriers) }\end{array}$ & $\begin{array}{c}\text { Novel COL4A2 } \\
\text { mutation } \\
\text { (2 carriers) }\end{array}$ \\
\hline \multicolumn{5}{|l|}{ Muscular abnormalities } \\
\hline Muscle cramps & 18 & & 2 & \\
\hline Myopathy & 2 & & 2 & \\
\hline Raynaud & 6 & & & \\
\hline Cardiac (supraventricular) arrhythmia & 4 & & 1 & \\
\hline Mitral valve prolapsed & 4 & & & \\
\hline Ventricular deptal defect & 1 & & & \\
\hline \multicolumn{5}{|l|}{ Other findings } \\
\hline
\end{tabular}

aNumbers indicate patients reported in the literature and in present cohort.

porencephaly; in 5 patients (A-II.1, D-II.1, E-II.1, G-II.1, H-II.1) this was already seen prenatally (in 2 of them, the COL4A1 mutation also was identified prenatally), and 6 patients (B-I.1 and B-II.1,J-II.1, J-II.1, K-II.1, M-II.1) were diagnosed after birth and showed hemi- or tetraplegia and/or seizures. Although most patients with an antenatal diagnosis presented after 30 weeks of pregnancy, one patient (D-II.1) presented at 25 weeks.

Of interest, in patient A-II.1 the porencephalic cyst showed enlargement on MRI of the brain in his first year of life. Patient D-II.1 developed severe hydrocephalus secondary to the hemorrhage. Recurrence of cerebral hemorrhage was documented in patient E-II.1, who presented with antenatal cerebral hemorrhage and porencephaly on antenatal brain MRI. She developed a severe hypoxic-ischemic encephalopathy, for which she received cooling therapy; she died soon after birth. Postmortem examination showed a novel parenchymal hemorrhage (Figure $2 \mathrm{~m}, \mathrm{n})$. Patient II.2 from family $\mathrm{C}$ had a congenital tetraplegia; however, data on brain imaging are lacking.

\section{Sporadic extensive bilateral porencephaly resembling hydranencephaly}

Hydranencephaly is defined as the end result of massive hemispheric necrosis and extreme ventricular dilation, with most of the hemispheres replaced by a cerebrospinal fluid-filled membranous sac, and relative preservation of the diencephalic and posterior cranial fossa brain structures, with a variable onset, even starting in the first trimester. ${ }^{41}$ In our cohort, patient II.1 from family $\mathrm{H}$ showed severe brain destruction with undetectable medial cerebral arteries, resembling hydranencephaly, at 33 weeks' gestation. The child was stillborn at 34 weeks of gestational age.

\section{Periventricular leukomalacia with intracranial calcification}

Periventricular leukomalacia (PVL) is defined as posthypoxic-ischemic leukoencephalopathy resulting from a pre- or perinatal hypoxic-ischemic insult. ${ }^{42} \mathrm{PVL}$ is characterized by focal periventricular necrosis and gliosis in the surrounding white matter. ${ }^{43}$ Intracranial calcifications also are reported in this context. ${ }^{14}$ In our cohort, patients A-I.2 and C-II.1 both show PVL without porencephaly (Figure $2 \mathrm{~b}, \mathrm{e}$ ). Brain calcifications were not present. Additional features in patient A-I.2 were congenital cataracts. Patient C-II.1 also showed ophthalmological features comprising microcornea, congenital cataract, and posterior embryotoxon. This combination, together with the family history, suggested the diagnosis in both patients.

\section{Axenfeld Rieger anomaly with leukoencephalopathy}

Axenfeld Rieger anomaly comprises a constellation of ocular findings affecting the anterior chamber, including the anterior chamber angle and aqueous drainage structures (iridogoniodysgenesis), iris hypoplasia, eccentric pupil, iris tears, and iridocorneal tissue adhesions traversing the anterior chamber. A frequent association consists of a posterior embryotoxon and a high risk of glaucoma and secondary blindness. In our newly reported family $\mathrm{C}$, the three individuals with the COL4A1 change (C-I.2, C-II.1, C-II.2) were affected with similar ophthalmological findings from the Axenfeld Rieger spectrum: cataract and microcornea. The neurological symptoms, however, varied greatly, from absence in patient C-I.2, to PVL and normal cognition in patient C-II.1, to severe mental retardation and spastic hemiplegia in patient C-II.2.

\section{Cortical malformations: schizencephaly}

Although cortical lesions secondary to tissue necrosis have been described in COL4A1 mutations, ${ }^{15}$ malformations of the cortex, in particular schizencephaly, have only been appreciated recently as being associated with COL4A1 mutations, ${ }^{37,39}$ Schizencephaly is defined as a cleft extending from the pial surface to the lateral ventricle, lined by heterotopic gray matter. ${ }^{44}$ 

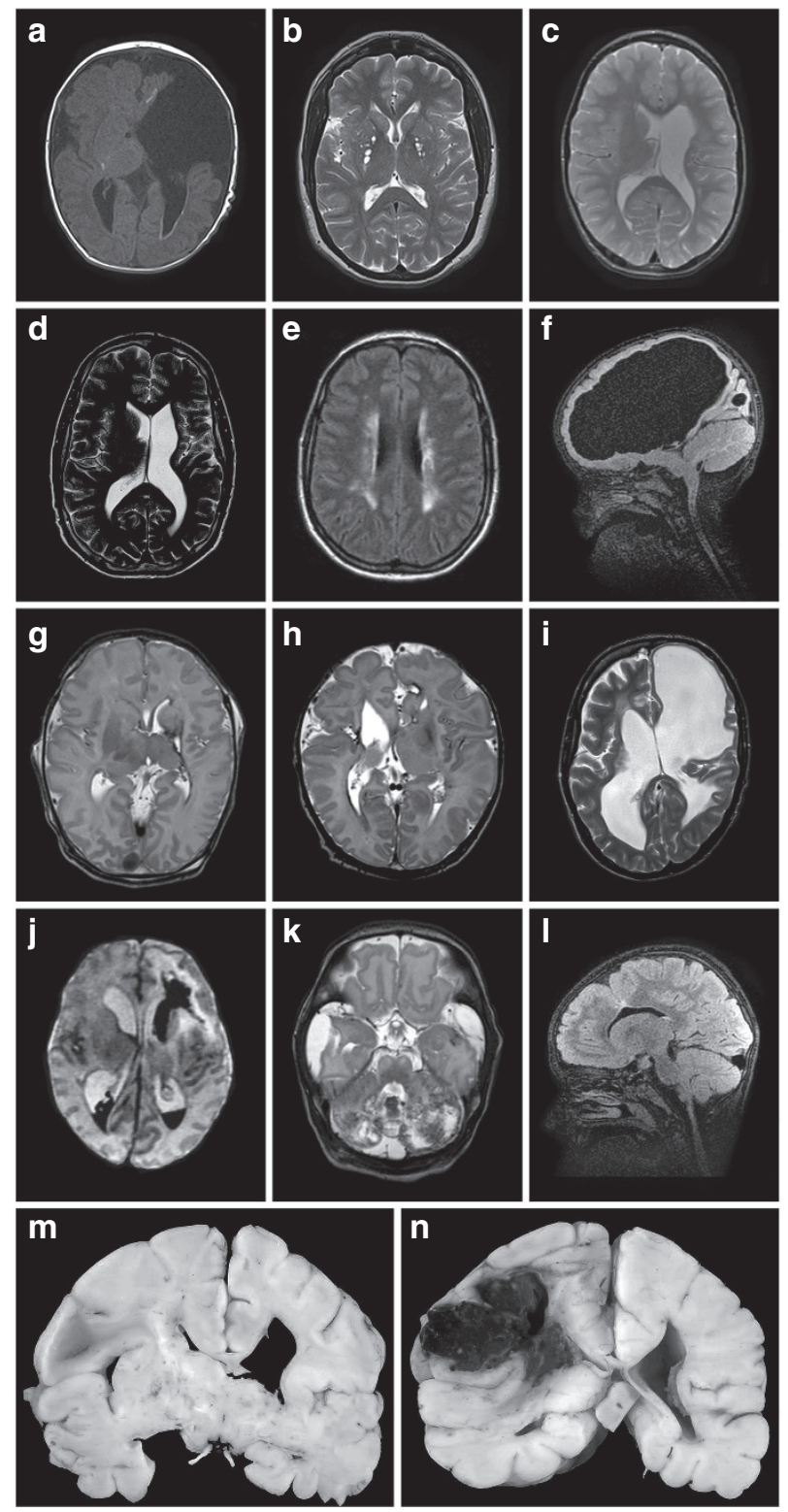

Figure 2 Selection of brain magnetic resonance images of novel patients. (a) T1-weighted image of patient A-ll.1 showing porencephaly with cortical destruction and white matter hyperintensities suggesting calcifications and/or hemorrhage. (b) T2-weighted image of patient A-I.2 showing enlarged perivascular spaces. (c,d) T2-weighed images of patients B-III.1 and B-II.1, with classic left-sided unilateral porencephaly. (e) Fluid-attenuated inversion recovery (FLAIR) image of patient C-II.1, indicating periventricular leukomalacia. (f) FLAIR image of patient D-II.1 depicting severe expansive porencephaly and severe white matter loss. (g) T2-weighed image of patient E-II.1 with leftsided hemorrhage with ipsilateral volume loss of white matter, thalamus, and basal ganglia. (h) T2-weighted image of patient H-Il.1 showing right-sided schizencephaly and porencephaly and a small hemorrhage in the left frontal lobe. (i) T2-weighted image of patient J-II. 1 with left-sided porencephaly with bilateral ventricular enlargement and leukoencephalopathy. (j) T2-weighted image of patient K-II. 1 with a left-sided germinal matrix hemorrhage leading to intraventricular hemorrhage and a left-sided venous infarction. (k) T2-weighted image of patient L-II.2 depicting bilateral severe cerebellar hemorrhage in both hemispheres. (I) FLAIR image of patient M-II.2 showing closed-lip schizencephaly and focal cortical dysplasia. $(\mathbf{m}, \mathbf{n})$ Postmortem images of patient E-II., showing ventricular dilatation and a large intraparenchymal hemorrhage.
In our cohort we observed two patients with focal cortical malformations. The first has a maternally inherited c.1964G $>$ A (p.G655E) COL4A1 mutation and presented with schizencephaly, porencephaly, and intraventricular hemorrhage (patient H-II.1; Figure 2h). The mother has a severe hypermetropia of +10 diopter. The second patient (L-II.1) has a paternally inherited c.3368A $>\mathrm{G}$ (p.E1123G) COL4A2 mutation and presented with porencephaly of the left frontal ventricle and an overlying dysplastic cortex resembling transmantle heterotopia on brain MRI (Figure 21). The father is asymptomatic, except for ptosis. The same COL4A2 mutation was previously reported in two adult patients with intraparenchymal hemorrhage; the mutation was proven to be pathogenic in in vitro functional studies. ${ }^{26}$

\section{Previously unreported findings in our cohort}

Patient II.1 from family K had a severe neonatal neurological presentation with seizures caused by intracerebral hemorrhages. At the age of 6 months he was diagnosed with neuroblastoma, which has not been previously associated with COL4A1 mutations but is a relatively frequent tumor in childhood. We mention this because of the occurrence of other neurological tumors in this cohort (meningioma in patient A-1.2 and in another COL4A1 carrier). ${ }^{7}$ Neural tumors as part of the phenotypic spectrum does not seem likely based on these data, but certainty remains unclear.

The index patient from family J presented with a left-sided germinal matrix and intraventricular hemorrhage and posthemorrhagic venous infarction after birth. Interestingly, supraventricular tachycardia also was noted at this young age, requiring sotalol treatment. Whether the tachycardia is causally related to the COL4A1 mutation is unclear; however, it has also been reported in hereditary angiopathy with nephropathy, aneurysm, and muscle cramps (HANAC) syndrome. ${ }^{16}$

\section{DISCUSSION}

In this cohort, 183 index patients were tested. Patients were referred from different hospitals in the Netherlands and other European countries. For most of the patients, clinical data at referral were provided and mostly included brain imaging evidence of porencephaly or infantile hemorrhage. In total, we identified 21 COL4A1 and 3 COL4A2 pathogenic or likely pathogenic mutations. This suggests a high prevalence (13\%) of mutations in this patient population. In the total cohort, a high percentage of de novo mutations was identified $(42 \% ; 10 / 24)$.

When reviewing the literature, a total of 67 families with COL4A1 and COL4A2 mutations were described. Among these families, 33 mutations (50\%) were inherited; a de novo mutation was identified in 17 (25\%), and in 17 cases (25\%) the data on parental sequencing were lacking. These data support our finding that the de novo mutation rate in COL4A1 and COL4A2 is high.

The clinical data of 13 families are novel, and the 12 COL4A1 mutations have not been described previously. No clear genotype-phenotype correlation is present. In our novel families, a high percentage $(60 \% ; 12 / 20)$ of severe perinatal presentation 
was observed. In this respect, one must consider an ascertainment bias because the main indication for referral for COL4A1 and COL4A2 sequencing was porencephaly or infantile cerebral hemorrhage, that is, the best known phenotype of COL4A1 and COL4A2 mutations. Although the clinical phenotypes of our patients greatly overlap the phenotype previously reported in the literature, this study corroborates the phenotypic spectrum of the less commonly reported features (Table 2). We discuss the reported phenotypes in the literature in comparison with those in our patients in the following sections.

\section{Prenatal and neonatal intracerebral hemorrhage and porencephaly}

Porencephaly is one of the most frequently reported findings, described in 53 patients with COL4A1 mutations ${ }^{7,8,12,14,15,17,18,21,37-39}$ and 6 patients with COL4A2 mutation ${ }^{27,28}$ (Table 2). Because porencephaly was the first reported associated phenotype, however, there may be a bias in the inclusion of patients for COL4A1 and COL4A2 testing. Intraventricular hemorrhage is a common complication of preterm infants with a very low birth weight, and a group of 40 preterm infants was tested for the presence of COL4A1 mutations. Only one pathogenic mutation was identified. ${ }^{10}$ This result indicates that COL4A1 mutations are probably only minor contributors to intraventricular hemorrhage in preterm infants with a very low birth weight. A recent report of COL4A1 screening in a group of 61 patients with porencephaly revealed mutations in 10 patients (16\%) aged 3 months to 14 years. All these patients had additional features including focal cortical dysplasia, intracranial calcification, hemolytic anemia, elevated creatine kinase, myopathy, ophthalmological features, or hematuria. ${ }^{39}$ A complication of the hemorrhage can be the development of secondary hydrocephalus, as seen in patient D-II.1 and previously reported. ${ }^{13,15,45}$

\section{Sporadic extensive bilateral porencephaly resembling hydranencephaly}

We previously described a subset of patients with extensive prenatal porencephaly and gray and white matter loss, cortical destruction, and cerebellar and brain stem atrophy, resembling hydranencephaly. A pathogenic mechanism, consisting of a massive germinal matrix hemorrhage followed by extensive venous infarction with compression, edema, and secondary ischemia of larger parenchymal areas and leading to white matter and cortical destruction, was suggested. Interestingly, we found de novo COL4A1 mutations in all these patients, including a case of germ-line mosaicism in the mother of one patient. ${ }^{15}$

\section{PVL with intracranial calcification}

Subtle periventricular, basal ganglia, and/or deep white matter calcifications are reported in COL4A1 mutations, together with PVL, also in the absence of porencephaly. ${ }^{14,33,37,39,46}$ The diagnosis in patients with this PVL phenotype without a contributing family history or additional findings could easily be missed. Associated features, such as elevated creatine kinase concentration or microbleeds, may help in suspecting the diagnosis in the absence of other major findings such as porencephaly. ${ }^{14,33,37,39}$ These associated findings may also help in discriminating COL4A1-related calcifications and leukoencephalopathy from disorders with partially overlapping manifestations, such as Aicardi-Goutières syndrome, classically characterized by infantile encephalopathy, cerebral calcifications, cerebral atrophy, and leukodystrophic white matter changes, ${ }^{47}$ as well as cytomegalovirus infection or cystic leukoencephalopathy, without megalencephaly. ${ }^{14,48}$

\section{Axenfeld Rieger anomaly with leukoencephalopathy}

Axenfeld Rieger anomaly, as well as cataracts, microcornea, and retinal detachment, have been reported as consistent findings in several other families harboring COL4A1 mutations. Although major cerebral findings such as porencephaly or hemorrhagic stroke can be present, all patients showed leukoencephalopathy and small-vessel disease, even in the absence of neurological symptoms. This indicates that brain MRI in patients with Axenfeld Rieger anomaly may provide a clue for the diagnosis of a COL4A1-related disorder. ${ }^{11,18}$

\section{HANAC syndrome}

The HANAC syndrome comprises a specific combination of features and is attributed to mutations in COL4A1 affecting glycine residues in close proximity of exons 24 and 25, reported to be the triple helical CB3[IV] domain, encompassing major integrin binding sites. ${ }^{16,32,35}$ The angiopathy in HANAC syndrome comprises retinal vessel arterial tortuosity and cerebral small- and large-vessel disease with aneurysms of the carotid syphon. The nephropathy consists of persistent hematuria and/ or proteinuria with or without bilateral large renal cysts. Other associated findings are muscle cramps with elevated creatine kinase concentrations, Raynaud phenomenon, and cardiac arrhythmia. Interestingly, patients with HANAC syndrome do not present with infantile hemiplegia or porencephaly. Also, the risk of major (hemorrhagic) stroke is thought to be lower than in other COL4A1 mutations. ${ }^{16,32,35}$ In our cohort, no patients displaying the HANAC phenotype were identified, possibly because of referral bias, since our cohort comprises mostly neurological patients. The clinical picture of HANAC is possibly insufficiently known by referring physicians to be associated with COL4A1 mutations.

\section{Stroke in childhood and young adulthood}

In addition to prenatal hemorrhage leading to porencephaly, strokes in the form of cerebral bleeding at a later age can occur with COL4A1 and COL4A2 mutations. ${ }^{17,20,31,34}$ Stroke in later childhood and young adulthood was not reported in our cohort. In the literature, cerebral hemorrhages in childhood or in young adulthood have been documented in patients with COL4A1 mutations. Differential diagnostic considerations in these cases comprise other genetic cerebrovascular malformation syndromes, such as cerebral cavernous hemangiomas or hereditary hemorrhagic telangiectasia. Hemorrhagic strokes 
typically affect the deep white matter, in combination with diffuse leukoencephalopathy and microbleeds. ${ }^{17,20,31,34}$ Recurrence of strokes has been reported. ${ }^{20,31}$ Two of the documented cases were sporadic, ${ }^{20,34}$ the others were familial..$^{17,31}$

\section{Sporadic late-onset hemorrhagic stroke}

Sporadic intracerebral hemorrhage generally occurs in the elderly, with a worldwide incidence of 24.6 per 100.000 personyears ${ }^{49}$ frequently in the setting of risk factors such as cerebral amyloid angiopathy or hypertensive vasculopathy, alcohol consumption, or cigarette smoking.

In our cohort, patients with adult-onset cerebral hemorrhage are lacking. This may very well be due to selection bias, probably because most patients were referred by clinical geneticists and neonatologists because of pre- and perinatal hemorrhage or porencephaly.

In a cohort of 96 sporadic patients with intracerebral hemorrhage not caused by arteriovenous malformations, tumors, or impaired coagulation, COL4A1 and COL4A2 were tested, leading to the detection of two COL4A1 mutations in two patients and three COL4A2 mutations in four patients. ${ }^{22,26}$ These findings indicate that COL4A1 and COL4A2 mutations contribute to $\sim 6 \%$ of sporadic late-onset intracerebral hemorrhage. Because intracerebral hemorrhage constitutes $\sim 15 \%$ of all intracranial hemorrhages in this group of patients, the contribution of COL4A1 and COL4A2 mutations to the total group of intracranial hemorrhage is $\sim 1 \%$. Although the portion may seem small, the general prevalence of COL4A 1 and COL4A2 mutations may be quite high; intracerebral hemorrhage in the elderly is not a rare event. The mutations identified in the patients are missense mutations, which probably have a milder effect on collagen IV function, and not the frequently identified triple helical domain glycine changes. ${ }^{22,26}$ However, the pathogenicity of these milder missense mutations has only been tested in an in vitro expression system.

\section{Focal cortical dysplasia and schizencephaly}

In the literature, COL4A1 mutations were identified as the first genetic cause of schizencephaly ${ }^{37,39}$; mutations were found in 5 of 10 patients with schizencephaly who were tested. We describe the first patient with cortical dysplasia harboring a COL4A2 mutation (L-II.1). The localization of the cortical dysplasia and the association with an underlying porencephalic enlargement of the frontal part of the lateral ventricle in our patient suggest a causal relation between the COL4A2 mutation and the cortical malformation (Figure 21). Interestingly, the father also carries the mutation and is asymptomatic, but no MRI has been performed. The pathogenesis of this malformation is probably similar to that of the schizencephaly and hydranencephaly reported in COL4A1 mutations. Our findings indicate that COL4A2 mutations can also lead to dysplastic cortex, thereby broadening the COL4A2 phenotypic spectrum. The role of COL4A2 mutations in cortical dysplasia associated with porencephaly needs to be investigated in a larger patient cohort.

\section{Issues in genetic counseling and management of affected families}

There are several plausible, non-mutually exclusive pathogenic mechanisms of various COL4A1 and COL4A2 mutations, which were summarized in a recent review..$^{25}$ The pathogenic mechanism is still not elucidated completely and may be different depending on mutation type.

Another unresolved question is the matter of the reduced penetrance. The same mutation can lead to a severely affected infant, while the carrier parent is hardly affected. This leads to the hypothesis that COL4A1 and COL4A2 mutations must be regarded as risk factors that, together with additional modifying factors, lead to a phenotype. Mouse models support this hypothesis, showing that penetrance and disease severity is related to the genetic context. ${ }^{13,50}$

Because of the reduced penetrance with possible modifying factors and the variable phenotype, the counseling of affected patients and their families remains a challenge. We suggest an initial workup in families with a mutation, including neurological, ophthalmological, renal, and cardiac screening in mutation carriers and first-degree relatives with a 50\% chance of harboring the mutation (the latter taking into account the social consequences of presymptomatic DNA testing) (Table 3). Neurological screening should include a medical history and physical examination, followed by brain MRI if abnormalities are found. We recommend adding susceptibility-weighted imaging because it improves the detection of microbleeds. ${ }^{51}$ Performing magnetic resonance angiography to identify cerebral aneurysms is still under debate; to date no ruptured aneurysms have been reported in mutation carriers, suggesting a slow progression rate and discouraging the use of magnetic resonance angiography. Follow-up data on the course of aneurysms identified in this specific group of patients, however, are lacking.

Another subject under debate is the perinatal management of pregnancies in which the child or the mother harbors a COL4A1 or COL4A2 mutation. Prenatal testing can be offered in highrisk pregnancies after genetic counseling, with special attention given to the variable phenotypic expression and reduced penetrance. Caesarean delivery has been proposed to prevent brain vascular injury attributable to birth trauma. ${ }^{30}$ Evidence that this will lead to the prevention of cerebral hemorrhage is lacking, however, and several patients who had lesions established long before delivery have now been reported. ${ }^{12,15,21,29,36}$ Further studies

\section{Table 3 Screening protocol at diagnosis}

Neurological examination

Brain magnetic resonance imaging (when indicated)

Ophthalmological examination

Renal ultrasound

Urine analysis for the presence of hematuria

Renal function test (serum creatinine, estimated glomerular filtration rate)

Serum creatine kinase measurement

Electrocardiogram (arrhythmias) 
of the clinical course of individuals with COL4A1 or COL4A2 mutations are needed to determine the follow-up in more detail.

\section{Conclusions}

Our data confirm that COLAA1 and COL4A2 mutations are important causes of cerebrovascular disease with a high mutation detection rate in porencephaly and childhood cerebral hemorrhage with a relatively high rate of de novo mutations. Although also present in (sporadic) adult-onset intracerebral hemorrhage, with an incidence of $6 \%, 22,26$ the role of mutations in this patient cohort seems less prominent. It seems important to increase awareness of this disorder among neurologists and internists coping with the adult population. In addition to the cerebrovascular phenotype, systemic involvement with ocular, renal, muscular, and cardiac features must not be underestimated and needs to be screened at diagnosis, even outside the formal HANAC syndrome. The precise role of COL4A1 and COL4A2 mutations in cortical malformations needs to be elucidated, but it seems to contribute to those malformations that are most likely a result of a vascular insult during fetal development. ${ }^{52}$ Follow-up data on COL4A1 and COL4A2 mutation carriers are important to develop appropriate surveillance protocols and adapt treatment.

\section{SUPPLEMENTARY MATERIAL}

Supplementary material is linked to the online version of the paper at http://www.nature.com/gim

\section{DISCLOSURE}

The authors declare no conflict of interest.

\section{ACKNOWLEDGMENTS}

This work was financially supported by Fonds NutsOhra.

\section{REFERENCES}

1. Zonana J, Adornato BT, Glass ST, Webb MJ. Familial porencephaly and congenital hemiplegia. J Pediatr 1986;109:671-674.

2. Sensi A, Cerruti S, Calzolari E, Vesce F. Familial porencephaly. Clin Genet 1990;38:396-397.

3. Berg RA, Aleck KA, Kaplan AM. Familial porencephaly. Arch Neurol 1983:40:567-569.

4. Shastri NJ, Bharani SA, Modi UJ, Trivedi C. Familial porencephaly. Indian J Pediatr 1993;60:459-463.

5. Mancini GM, de Coo IF, Lequin MH, Arts WF. Hereditary porencephaly: clinical and MRI findings in two Dutch families. Eur J Paediatr Neurol 2004;8:45-54.

6. Vilain C, Van Regemorter N, Verloes A, David P, Van Bogaert P. Neuroimaging fails to identify asymptomatic carriers of familial porencephaly. Am J Med Genet 2002;112:198-202.

7. Breedveld $\mathrm{G}$, de Coo IF, Lequin $\mathrm{MH}$, et al. Novel mutations in three families confirm a major role of COL4A1 in hereditary porencephaly. J Med Genet 2006:43:490-495.

8. Gould DB, Phalan FC, Breedveld GJ, et al. Mutations in Col4a1 cause perinatal cerebral hemorrhage and porencephaly. Science 2005; 308:1167-1171.

9. Aguglia U, Gambardella A, Breedveld GJ, et al. Suggestive evidence for linkage to chromosome 13qter for autosomal dominant type 1 porencephaly. Neurology 2004;62:1613-1615

10. Bilguvar K, DiLuna ML, Bizzarro MJ, et al.; Pacifier and Breastfeeding Trial Group. COL4A1 mutation in preterm intraventricular hemorrhage. J Pediatr 2009;155:743-745.
11. Coupry I, Sibon I, Mortemousque B, Rouanet F, Mine M, Goizet C. Ophthalmological features associated with COL4A1 mutations. Arch Ophthalmol 2010;128:483-489.

12. de Vries LS, Koopman C, Groenendaal F, et al. COL4A1 mutation in two preterm siblings with antenatal onset of parenchymal hemorrhage. Ann Neurol 2009;65:12-18.

13. Labelle-Dumais C, Dilworth DJ, Harrington EP, et al. COL4A1 mutations cause ocular dysgenesis, neuronal localization defects, and myopathy in mice and Walker-Warburg syndrome in humans. PLoS Genet 2011;7:e1002062.

14. Livingston J, Doherty D, Orcesi S, et al. COL4A1 mutations associated with a characteristic pattern of intracranial calcification. Neuropediatrics 2011;42:227-233.

15. Meuwissen ME, de Vries LS, Verbeek HA, et al. Sporadic COL4A1 mutations with extensive prenatal porencephaly resembling hydranencephaly. Neurology 2011;76:844-846.

16. Plaisier E, Gribouval O, Alamowitch S, et al. COL4A1 mutations and hereditary angiopathy, nephropathy, aneurysms, and muscle cramps. N Engl I Med 2007;357:2687-2695.

17. Shah S, Ellard S, Kneen R, et al. Childhood presentation of COL4A1 mutations. Dev Med Child Neurol 2012;54:569-574.

18. Sibon I, Coupry I, Menegon P, et al. COL4A1 mutation in Axenfeld-Rieger anomaly with leukoencephalopathy and stroke. Ann Neurol 2007;62: 177-184.

19. Vahedi K, Boukobza M, Massin P, Gould DB, Tournier-Lasserve E, Bousser MG. Clinical and brain MRI follow-up study of a family with COL4A1 mutation. Neurology 2007;69:1564-1568.

20. Vahedi K, Kubis N, Boukobza M, et al. COL4A1 mutation in a patient with sporadic, recurrent intracerebral hemorrhage. Stroke 2007;38:1461-1464.

21. Vermeulen RJ, Peeters-Scholte C, Van Vugt JJ, et al. Fetal origin of brain damage in 2 infants with a COL4A1 mutation: fetal and neonatal MRI. Neuropediatrics 2012;42:1-3.

22. Weng YC, Sonni A, Labelle-Dumais $C$, et al. COL4A1 mutations in patients with sporadic late-onset intracerebral hemorrhage. Ann Neurol 2012;71:470-477.

23. Khoshnoodi J, Pedchenko V, Hudson BG. Mammalian collagen IV. Microsc Res Tech 2008;71:357-370.

24. Khoshnoodi J, Cartailler JP, Alvares K, Veis A, Hudson BG. Molecular recognition in the assembly of collagens: terminal noncollagenous domains are key recognition modules in the formation of triple helical protomers. J Biol Chem 2006;281:38117-38121.

25. Kuo DS, Labelle-Dumais C, Gould DB. COL4A1 and COL4A2 mutations and disease: insights into pathogenic mechanisms and potential therapeutic targets. Hum Mol Genet 2012;21(R1):R97-110.

26. Jeanne $M$, Labelle-Dumais $C$, Jorgensen J, et al. COL4A2 mutations impair COL4A1 and COL4A2 secretion and cause hemorrhagic stroke. Am J Hum Genet 2012;90:91-101

27. Verbeek E, Meuwissen ME, Verheijen FW, et al. COL4A2 mutation associated with familial porencephaly and small-vessel disease. Eur J Hum Genet 2012;20:844-851.

28. Yoneda $\mathrm{Y}$, Haginoya $\mathrm{K}$, Arai $\mathrm{H}$, et al. De novo and inherited mutations in COL4A2, encoding the type IV collagen a2 chain cause porencephaly. Am J Hum Genet 2012;90:86-90.

29. Lichtenbelt KD, Pistorius LR, De Tollenaer SM, Mancini GM, De Vries LS. Prenatal genetic confirmation of a COL4A1 mutation presenting with sonographic fetal intracranial hemorrhage. Ultrasound Obstet Gynecol 2012;39:726-727.

30. Gould DB, Phalan FC, van Mil SE, et al. Role of COL4A1 in small-vessel disease and hemorrhagic stroke. N Eng/ J Med 2006;354:1489-1496.

31. Shah $S$, Kumar Y, McLean B, et al. A dominantly inherited mutation in collagen IV A1 (COL4A1) causing childhood onset stroke without porencephaly. Eur J Paediatr Neuro/ 2010;14:182-187.

32. Alamowitch S, Plaisier E, Favrole P, et al. Cerebrovascular disease related to COL4A1 mutations in HANAC syndrome. Neurology 2009;73:1873-1882.

33. Rouaud T, Labauge $P$, Tournier Lasserve $E$, et al. Acute urinary retention due to a novel collagen COL4A1 mutation. Neurology 2010;75:747-749.

34. Coutts SB, Matysiak-Scholze U, Kohlhase J, Innes AM. Intracerebral hemorrhage in a young man. CMAJ 2011;183:E61-E64.

35. Plaisier E, Chen Z, Gekeler F, et al. Novel COL4A1 mutations associated with HANAC syndrome: a role for the triple helical CB3[IV] domain. Am J Med Genet A 2010;152A:2550-2555.

36. Garel C, Rosenblatt J, Moutard ML, et al. Fetal intracerebral hemorrhage and COL4A1 mutation: promise and uncertainty. Ultrasound Obstet Gynecol 2012;41:228-230. 
37. Tonduti D, Pichiecchio A, La Piana $R$, et al. COL4A1-related disease: raised creatine kinase and cerebral calcification as useful pointers. Neuropediatrics 2012:43:283-288.

38. Lemmens R, Maugeri A, Niessen HW, et al. Novel COL4A1 mutations cause cerebral small vessel disease by haploinsufficiency. Hum Mol Genet 2013:22:391-397.

39. Yoneda $Y$, Haginoya K, Kato M, et al. Phenotypic spectrum of COL4A1 mutations: porencephaly to schizencephaly. Ann Neurol 2013;73:48-57.

40. Rødahl E, Knappskog PM, Majewski J, et al. Variants of anterior segment dysgenesis and cerebral involvement in a large family with a novel COL4A1 mutation. Am J Ophthalmol 2013;155:946-953.

41. Cecchetto G, Milanese L, Giordano R, Viero A, Suma V, Manara R. Looking at the missing brain: hydranencephaly case series and literature review. Pediatr Neurol 2013:48:152-158.

42. van der Knaap MS, Valk J, de Neeling N, Nauta JJ. Pattern recognition in magnetic resonance imaging of white matter disorders in children and young adults. Neuroradiology 1991;33:478-493.

43. Nagasunder AC, Kinney HC, Blüml S, et al. Abnormal microstructure of the atrophic thalamus in preterm survivors with periventricular leukomalacia. AJNR Am J Neuroradio/ 2011:32:185-191.

44. Dies KA, Bodell A, Hisama FM, et al. Schizencephaly: association with young maternal age, alcohol use, and lack of prenatal care. J Child Neurol 2013;28:198-203.
45. van der Knaap MS, Smit LM, Barkhof F, et al. Neonatal porencephaly and adult stroke related to mutations in collagen IV A1. Ann Neuro/ 2006;59:504-511.

46. Livingston JH, Stivaros S, van der Knaap MS, Crow YJ. Recognizable phenotypes associated with intracranial calcification. Dev Med Child Neurol 2013;55:46-57.

47. Crow YJ, Rehwinkel J. Aicardi-Goutieres syndrome and related phenotypes: linking nucleic acid metabolism with autoimmunity. Hum Mol Genet 2009;18(R2):R130-R136.

48. Henneke M, Diekmann S, Ohlenbusch A, et al. RNASET2-deficient cystic leukoencephalopathy resembles congenital cytomegalovirus brain infection. Nat Genet 2009;41:773-775.

49. van Asch CJ, Luitse MJ, Rinkel GJ, van der Tweel I, Algra A, Klijn CJ. Incidence, case fatality, and functional outcome of intracerebral haemorrhage over time, according to age, sex, and ethnic origin: a systematic review and meta-analysis. Lancet Neurol 2010;9:167-176.

50. Gould DB, Marchant JK, Savinova OV, Smith RS, John SW. Col4a1 mutation causes endoplasmic reticulum stress and genetically modifiable ocular dysgenesis. Hum Mol Genet 2007;16:798-807.

51. Cheng AL, Batool S, McCreary CR, et al. Susceptibility-weighted imaging is more reliable than $\mathrm{T} 2{ }^{*}$-weighted gradient-recalled echo MRI for detecting microbleeds. Stroke 2013;44:2782-2786.

52. Robin NH, Taylor CJ, McDonald-McGinn DM, et al. Polymicrogyria and deletion 22q11.2 syndrome: window to the etiology of a common cortical malformation. Am J Med Genet A 2006;140:2416-2425. 